\title{
$\nabla$ \\ Recent Trends in Sensitivity Pattern of Staphylococcus species
}

\section{IJCRR}

Section: Healthcare

ISI Impact Factor

(2019-20): 1.628

IC Value (2019): 90.81

$\operatorname{SJIF}(2020)=7.893$

(c) (i) (8)

Copyright@IJCRR

\section{Otta Sarita ${ }^{1}$, Bhoi Priyadarshini ${ }^{2}$, Sahu Kundan Kumar ${ }^{3}$, Swain Bichitrananda ${ }^{4}$}

'MD (Microbiology), Associate Professor, 1MS and SUM Hospital, Bhubaneswar, Odisha, lndia; ${ }^{2} \mathrm{MD}$ (Microbiology), Assistant Professor, $1 \mathrm{MS}$ and SUM Hospital, Bhubaneswar, Odisha, India; ${ }^{3} \mathrm{MD}$ (Microbiology), Professor and Head, IMS and SUM Hospital, Bhubaneswar, Odisha, India; ${ }^{4} \mathrm{MD}$ (Microbiology), Associate Professor, SLN Medical College and Hospital, Koraput, Odisha, India.

\section{ABSTRACT}

Introduction: Staphylococcus particularly MRSA (Methicilin resistant Staphylococcus aureus) and MR CoNS (Methicilin resistant Coagulase Negative Staphylococcus) are important cause of hospital and community acquired infections. But there are reports of resistance to anti-MRSA drugs particularly vancomycin, teicoplanin, linezolid, tigecyclin which is a cause forconcern.

Aim: The present study was conducted to determine the susceptibility of Staphylococci and note the pattern of minimum inhibitory concentration (MIC) of the second line anti staphylococcal drugs in common clinical use.

Method: Various culture samples submitted to Department of Microbiology from Sept 2019 to May 2020 and yielding growth of Staphylococcus were included in the present study. Sensitivity pattern was determined by Vitek 2, Biomerioux and MIC of drugs were interpreted as per recent guidelines.

Result: 264 Staphylococcus were included in the study of which 39 were Staphylococcus aureus, 72 were S. epidermidis and 67 were S. haemolyticus.Besides these, 46, 14 and 11 strains of S.hominis, $S$ cohinii and S. capitis respectively were isolated. With cefoxitin screen method, $23(58.9 \%)$ isolates were MRSA where asonly $16(41.03 \%)$ were MSSA. Of the S.epidermidis and S.haemolyticus isolated $98.59 \%$ and $95.01 \%$ were methicillin resistant and all other isolated CONS were methicillin resistant. MRSA isolates had higher degree of resistance in comparison to MSSA to all the antibiotics tested except quinolones and erythromycin.All the S.aureus isolates were $100 \%$ sensitive to linezolid, vancomycin, daptomycin, teicoplanin, rifampicin and nitrofurantoin. None of the Staphylococcus showed any creeping of the minimum inhibitory concentration (MIC)of vancomycin. CONS particularly S.epidermidis and S. haemolyticus had a greater degree of resistance to linezolid, daptomycin and teicoplanin than S.aureus. Tigecyclin a reserve drug was the most sensitive antibiotic in all the species.

Conclusion: The study highlights high degree of resistance in CONS isolates to all drugs. Linezolid a useful oral drug also showed high resistance to many CONS and few S.aureus strains. Rifampicin and tigecyclin are other useful drugs for Staphylococcus in the present study.

Keywords: Staphylococcus spp, Vitek 2, Linezolid resistance, Tigecycline, Vancomycin, MIC creep.

\section{INTRODUCTION}

Staphylococcus particularly MRSA (Methicilin resistant Staphylococcus aureus) and MR CONS (Methicilin resistant Coagulase Negative Staphylococcus) are important cause of nosocomial and community acquired infections ranging from mild localized skin and appendage infections to life threatening conditions such as sepsis, necrotizing pneumonia, septic arthritis, endocarditis, and osteomyelitis. ${ }^{1,2}$ CONS a normal commensal of skin and mucous membrane is recently being implicated as an important nosocomial pathogen particularly when same strain is repeatedly isolated from same site. ${ }^{3}$ Vancomycin is the treatment of choice for serious MRSA infections. ${ }^{4}$ In the last few years, drugs such as linezolid, daptomycin, tigecycline, dalbavancin, telavancin, oritavancin, ceftobiprole, ceftaroline, and iclaprim have been added to the arsenal of anti-MRSA drugs. ${ }^{5}$ But few reports suggest resistance to even these alternative drugs which is troublesome and necessitates continuous monitoring. The present study was conducted to determine the susceptibility of Staphylococci to the various antibiotics and note the pattern of MIC to the second line anti staphylococcal drugs

\section{Corresponding Author:}

Dr. Sarita Otta, 1/5, Kanchanjunga Enclaves, Chandrasekharpur, Bhubaneswar, Odisha, India.

Phone: 09438529628; Email: saritaotta@gmail.com

ISSN: 2231-2196 (Print) ISSN: 0975-5241 (Online)

Received: $23.01 .2021 \quad$ Revised: 05.03 .2021

Accepted: 10.04 .2021

Published: 26.05.2021 
in common clinical use i.e vancomycin (glycopeptide), daptomycin (lipopeptide), tigecycline (glycylcycline) and linezolid (oxazolidinone).

\section{MATERIALS AND METHODS}

Various culture samples (blood, wound swab, pus, urine etc) submitted to Department of Microbiology, IMS and SUM Hospital, Bhubaneswar from Sept 2019 to May 2020 were included in the present study. Blood samples were processed by automated blood culture system (bio Mérieux). All positive blood cultures and other samples were streaked on blood agar and Macconkey agar plates. The colonies thus received on the plates after overnight incubation were submitted for Gram staining. The Gram positive cocci obtained were subjected to identification and susceptibility testing by Vitek 2 (bio Mérieux) automated system. In case of Staphylococcus other than S.aureusthe isolate was considered only when clinically significant. Minimum inhibitory concentration (MIC) was determined by Vitek 2 and the results were interpreted as per CLSI guidelines. (Table 1) For tigecycline interpretation was done as per European Committee On Antimicrobial Susceptibilty Testing(EUCAST) guidelines. (Table 1) Staphylococcus aureus ATCC 29213 was employed for quality control. Being a National Accreditation Board for Testing and Calibration Laboratories(NABL) accredited lab all procedures and chemicals were standardized and quality assured.

\section{RESULTS}

A total 264 Staphylococcus were isolated in the lab from non duplicate non repeat clinically significant samples during the study period. Among them 39 were Staphylococcus aureus, 72 were S.epidermidis and 67 were S.haemolyticus. Besides these, other less common species isolated were 46, 14 and 11 strains of $S$ hominis, $S$ cohinii and $S$ capitis respectively. With cefoxitin screen method, 23 (58.9\%) isolates were MRSA where asonly 16 (41.03\%) were MSSA. Of the total S.epidermidis and S.haemolyticus isolated $98.59 \%$ and $95.01 \%$ were methicillin resistant. All other CONS isolated were $100 \%$ cefoxitin screen positive. Inducible clindamycin resistance was highest in S.aureus strains than that for CONS being $37.5 \%$ in MRSA and $39.1 \%$ in MSSA varieties. (Table 2)

When the sensitivity pattern was looked into MRSA isolates had higher degree of resistance in comparison to MSSA to all the antibiotics tested except quinolones and erythromycin. All the S.aureus isolates were $100 \%$ sensitive to linezolid, vancomycin, daptomycin, teicoplanin, rifampicin and nitrofurantoin. Few MSSA isolates (4.4\%) strikingly showed resistance to tigecyclin. S.aureus isolates showed very low sensitivity to ciprofloxacin (13\% to MSSA and 25\% to MRSA), levofloxacin (13\% in MSSA and $18.75 \%$ in MRSA) and erythromycin (23.08\% in MSSA and $43.75 \%$ in MRSA). The MRSA isolates had additionally lower sensitivity to penicillin (13.3\%).(Table 3)

CONS had a higher degree of resistance thanS.aureusto almost all the antibiotic. Penicillin as expected is the least useful drug while vancomycin followed by tigecyclin is the most useful antibiotic.Nitrofurantoin is a good drug for all the species except $S$. haemolyticus. It is astonishing to find resistance to linezolid, teicoplanin and daptomycin in few CONS strains as these are the commonly reserved antibiotics for Staphylococcal strains. (Table 3)

S.epidermidis showed $100 \%$ sensitivity to vancomycin, tigecyclin and other drugs like rifampicin and nitrofurantoin. But higher degree of resistance was seen in these isolates to linezolid, teicoplanin and daptomycin in comparison to S.aureus. Quinolones are relatively more useful for the treatment of these strains but all other antibiotics show a higher degree of resistance than that of S.aureus. (Table 3)

S.haemolyticus isolates have a much higher degree of resistance to all the tested antibiotics among the different species of Staphylococcus. But vancomycin and tigecyclin are 100\% effective in vitro against all the strains.

The MIC of all the second-line drugs were compared. In our study, none of the isolates of Staphylococcus showed any creeping of the MIC values to suggest heteroresistant strains of Vancomycin. In the case of linezolid, there were 7 strains of S.epidermidis, 1 of S. haemolyticus. 2 isolates of $S$ cohinii and 1 of $S$ capitis showing MIC of 8 . Again 10 isolates of $S$ haemolyticus had a MIC $>8$. (Fig 1)

For Daptomycin, among the 4 resistant strains of S epidermidis, 2 isolates each had a MIC of 2 and 4.In the case of $S$. haemolyticus there were 1 strain each with MIC of 2, 4, 8 for Daptomycin. 2 strains had MIC 4 and 8 of $S$ hominis and 1 strain of S.capitis had MIC of 2. (Fig 1)

There were only 2 samples each of S.haemolyticus and S.hominis with MIC $>32$ for teicoplanin. For tigecycline, the majority of samples had a MIC of $<0.5$. Only one sample of S haemolyticus and 2 of S epidermidis had a MIC of 1 and 2 respectively for tigecycline. (Fig 1)

\section{DISCUSSION}

S.aureus developed penicillin resistance soon after its introduction and followed it with methicillin resistance as well. MRSA strains are not only resistant to almost all beta-lactams but have also developed resistance to multiple other antimicrobial classes. ${ }^{6}$ with the increasing prevalence of MRSA, the use of vancomycin for treatment of invasive infections 
has increased, along with concerns about vancomycin resistance among MRSA. ${ }^{7}$ CONS along with S.aureusis an important cause of nosocomial infection. The 30-day mortality of patients with CONS bacteraemia was up to $12.7 \%(20 / 157)$, particularly when associated with old age, Chronic liver and renal disease in a study. ${ }^{8}$ Thus knowing the recent pattern of resistance in the case of Staphylococcus is important not only for treatment but also to prevent the spread of infection.

In this study 39 S.aureuswhere as 225 CONS were isolated. Among the CONS most common species isolated was S.epidermidis and S.haemolyticus.Staphylococcus epidermidis is the most common colonizer of skin and mucous membranes and is the commonest cause of catheter-associated bloodstream infections. ${ }^{9}$ Other similar studies ${ }^{10}$ also noted S.epidermidis and S.haemolyticus as the most predominant species of CONS.

In the present study of all S.aureus, $41.03 \%$ were Methicillin-resistant while $96.4 \%$ of CONS were methicillin-resistant. The higher rate in later is due to their penicillinase production and these are resistant to all the beta-lactams except the recently developed ceftaroline. In a study at Aligarh in India, ${ }^{11}$ its was shown that $35.1 \%$ of $S$. aureus and $22.5 \%$ of coagulase-negative staphylococcal isolates were resistant to methicillin. Another study ${ }^{12}$ also found a higher rate of MR in S.aureus than CONS in contrast to our findings. Similar to the present study, Methicillin resistance is more common among $S$. epidermidis and $S$. haemolyticusisolates than among $S$. aureus in few other recent studies. ${ }^{13-15}$ CONS are often regarded as a harborer of resistant genes. Probably this suggests the requirement of more stringent infection control measures.

All the species showed a high degree of resistance to penicillin. Penicillin resistance presumes resistance to all other beta-lactam drugs as well. This is probably due to the abundant production of the penicillinase enzyme. In the present study both the quinolones used had a similar degrees of sensitivity except for MRSA where ciprofloxacin had better sensitivity than levofloxacin. S.aureus had a much lower sensitivity to quinolones than CONS. Among the CONS S. haemolyticus had the highest degree of resistance to quinolones as well as for macrolides. A high degreeof resistance to these drugs was noted by other studies also. ${ }^{8,16}$ Some recent studies suggested that CONS were highly resistant to penicillin $(94.7 \%)$, oxacillin(90.7\%), and erythromycin $(85.3 \%)$, and more than $50 \%$ were resistant to cephalosporins, aminoglycosides and quinolones. ${ }^{17,18}$ But cotrimoxazole had a better sensitivity in the present study than most other studies. ${ }^{8,16}$ Gentamicin in our study continues to have very good sensitivity to all the species of Staphylococcus similar to other studies ${ }^{8}$ although a report ${ }^{16}$ showed higher resistance.

Linezolid, a synthetic oxazolidinone having bacteriostatic activity against MRSA is rampantly used for the treatment of complicated skin and skin structure infections and nosocomial pneumonia. ${ }^{19}$ Present study noted that CONS particularly S.epidermidis and S. haemolyticus had a greater degree of resistance to this antibiotic than S.aureus. This is higher than that noted in other studies. ${ }^{8,10}$ Daptomycin and teicoplanin similarly had few CONS strains resistant to them. Tigecycline a reserve drug was the most sensitive antibiotic to all the species.

Rifampicinresistanceis more common inS. aureus than that for CONS. This antibiotic can penetrate biofilms, so is the antibiotic of choice for the treatment of bone and joint infections in both chronic and acute cases. ${ }^{20,21}$ In the present study the resistance noted to this antibiotic was only to a few of the CONS isolates. It is a reserve drug on our part leading to such a low degree of resistance.

Reports, even in India, have described MRSA and MSSA strains with vancomycin MICs increased to the high end of the Clinical and Laboratory Standards Institute (CLSI) susceptibility range i.e MIC creep. ${ }^{22-24}$ This is due to clonal replacement with cells containing subpopulations of cells in the vancomycin intermediate range (VISA, MIC 4-8 $\mu \mathrm{g} / \mathrm{ml}$ ) Few studies, also show $42 \%$ of Staphylococcus haemolyticus, a CONS, being intermediate resistant to vancomycin. ${ }^{25}$ Higher MIC of vancomycin is associated with an increased risk of treatment failure. ${ }^{6}$ In the present study no such heteroresistant Staphylococcus strains were found.

The present study carries limitations because of the short study period and taking Vitek MIC instead of actual E-test data for results. But this enlightens regarding the rising prevalence of resistance in S.aureus and CONS strains to the anti-A MRSA drugs and continued surveillance is a must to tackle it.

This is a lab-based study where samples were the routine cultures coming for reporting thus requiring no patient participation and raising no ethical concerns.

\section{CONCLUSION}

Rising prevalence of resistance to anti-A MRSA drugs to S.aureus is no new concept but the study highlights a high degree of resistance in CONS isolates to all drugs. Linezolid a useful oral drug also showed high resistance to many CONS and few S.aureus strains. As the CONS strains are harbourers and transmitters of resistance, this poses a challenge to antimicrobial stewardship measures in any hospital. None of the Staphylococcal isolates showed creeping MIC for vancomycin. Rifampicin and tigecycline are other useful drugs for Staphylococcus. 


\section{ACKNOWLEDGEMENT}

We acknowledge SOA University, Bhubaneswar, Odisha for their help in doing this work. The authors are also grateful to authors, editors, publishers of all those articles, journals and books from where the literature for this article has been reviewed and discussed

\section{Source of support: Nil}

\section{Conflict of interest: Nil}

\section{REFERENCES}

1. Boucher HW, Corey GR. Epidemiology of methicillin-resistant Staphylococcus aureus. Clin Infect Dis. 2008;46 (5):S344-9.

2. Valle DL Jr, Paclibare PA, Cabrera EC, Rivera WL. Molecular and phenotypic characterization of methicillin-resistant Staphylococcus aureus isolates from a tertiary hospital in the Philippines. Trop Med Health. 2016; 44:3.

3. Samad L, Kakru D K, Fonda B A, Roohi S, Lone M S, Ahmad J, et al.Prevalence and Risk Factors Associated with CoagulaseNegative Staphylococcus Infections in a Tertiary Care Center in North India. Int J Cur Res Rev 2017; 9(7): 39-43.

4. Koh YR, Kim KH, Chang CL, Yi J. Prevalence and clinical impact of heterogeneous vancomycin-intermediate Staphylococcus aureus isolated from hospitalized patients. Ann Lab Med 2016; $36: 235-43$.

5. NagasundaramN, Sujatha S. Worrisome trends in rising minimum inhibitory concentration values of antibiotics against methicillin-resistant Staphylococcus aureus - Insights from a tertiary care centre, South India. Braz J Infect Dis. 2015; 19(6): 585-89.

6. Diekema DJ, Pfaller MA, Turnidge J, Verhoef J, Bell J, Fluit $\mathrm{AC}$, et al.Genetic relatedness of multidrug-resistant, methicillin (oxacillin)-resistant Staphylococcus aureus bloodstream isolates from SENTRY Antimicrobial Resistance Surveillance Centers worldwide, 1998. Microb Drug Resist 2000; 6:213-21.

7. Diekema DJ, Richter SS, Heilmann KP, Dorhn CL, Riahi F, Tendolkar S et al. Continued emergence of USA 300 methicillin-resistant Staphylococcus aureus in the United States: results from a nationwide surveillance study. Infect Control Hosp Epidemiol. 2014; 35:285-92.

8. Cui J, Liang Z, Mo Z, Zhang J. The species distribution, antimicrobial resistance and risk factors for poor outcome of coagulase-negative staphylococci bacteraemia in China. AntimicrobResis Infect Contr. 2019; 8:65

9. Otto M.Staphylococcal infections: mechanisms of biofilm maturation and detachment as critical determinants of pathogenicity. Annu Rev Med. 2013; 64:175-88.

10. Al Tayyar I A, AL-Zoubi M S, Hussein E, Khudairat S, Sarosiekf K. Prevalence and antimicrobial susceptibility pattern of coagulase-negative staphylococci (CoNS) isolated from clinical specimens in Northern Jordan. Iran J Microbiol 2015:7(6):294301.

11. Dar JA, Thoker MA, Khan JA, Ali A, Khan MA, Rizwan M, et al. Molecular epidemiology of clinical and carrier strains of methicillin-resistant Staphylococcus aureus (MRSA) in the hos- pital settings of north India. Ann Clin Microbiol Antimicrob. 2006; 5:22.

12. Khadri H, Alzohairy M. Prevalence and antibiotic susceptibility pattern of methicillin-resistant and coagulase-negative staphylococci in a tertiary care hospital in India. Int J Med and Med Sc 2010; 2(4):116-20.

13. Garza-Gonzalez E, Morfin-Otero R, Llaca-Diaz JM, RodriguezNoriega E. Staphylococcal cassette chromosome mec(SCC mec) in methicillin-resistant coagulase-negative staphylococci. A review and the experience in a tertiary-care setting. Epidemiol Infect2010;138(5):645-54.

14. Barros EM, Ceotto H, Bastos MC, Dos Santos KR, GiambiagiDemarval M. Staphylococcus haemolyticusas an important hospital pathogen and carrier of methicillin resistance genes. J Clin Microbiol2012;50(1):166-8.

15. Mendes RE, Deshpande LM, Costello AJ, Farrell DJ. Molecular epidemiology of Staphylococcus epidermidis clinical isolates from US hospitals. Antimicrob Agents Chemother 2012; 56:4656-61.

16. Mohan U, Jindal N, Aggarwal P. Species distribution and antibiotic sensitivity pattern of coagulase-negative staphylococci isolated from various clinical specimens. Int J Med Microbiol 2002; 20(1): 45-46.

17. Asaad AM, Ansar Qureshi M, Mujeeb Hasan S. Clinical significance of coagulase-negative Staphylococci isolates from nosocomial bloodstream infections. Infect Dis (Lond). 2016; 48(5):356-60.

18. Shah MU, Akram MF, Usman J, Kaleem F. Incidence and susceptibility pattern of methicillin-resistant coagulase-negative Staphylococci isolated from a tertiary care hospital of Pakistan. Jundishapur J Microbiol. 2014; 7(1):e8590.

19. Scott TM. Alternatives to vancomycin for the treatment of methicillin-resistant Staphylococcus aureus infections. Clin Infect Dis. 2007; 45: S184-90.

20. Zimmerli W. Clinical presentation and treatment of orthopaedic implant-associated infection. J Intern Med.2014; 276(2):111-19.

21. Tuchscherer L, Kreis CA, Hoerr V, Flint L, Hachmeister M, Geraci J, et al. Staphylococcus aureus develops increased resistance to antibiotics by forming dynamic small colony variants during chronic osteomyelitis. J Antimicrob Chemother. 2016; 71(2):438-48.

22. Soriano A, Marco F, Martinez J, Pisos E, Almela M, DimovaV, et al. Influence of vancomycin minimum inhibitory concentration on the treatment of methicillin-resistant Staphylococcus aureus bacteremia. Clin Infect Dis. 2008; 46:193-200.

23. Di Gregorio S, Perazzi B, Ordonez AM, De Gregorio S, Foccoli $\mathrm{M}$, Lasala MB, et al. Clinical, microbiological, and genetic characteristics of heteroresistant vancomycin-intermediate Staphylococcus aureus bacteremia in a teaching hospital. Microb Drug Resist. 2015; 21(1):25-34.

24. Mohanty S, Behera B, Sahu S, Praharaj AK. The recent pattern of antibiotic resistance in Staphylococcus aureus clinical isolates in Eastern India and the emergence of reduced susceptibility to vancomycin. J Lab Phys. 2019; 11:340-45.

25. Srinivasan A, Dick JD, Perl TM. Vancomycin resistance in Staphylococci. Clin Microb Rev. 2002; 15(3): 430-8.

26. Jacob JT, Diaz Granados CA. High vancomycin minimum inhibitory concentration and clinical outcomes in adults with methicillin-resistant Staphylococcus aureus infections: ametaanalysis. Int J Infect Dis. 2013; 17:e93-100. 
Table 1: Interpretation criteria for various drugs

\begin{tabular}{lccccccccccccccccc} 
& \multicolumn{3}{c}{ Vancomycin } & \multicolumn{4}{c}{ Linezolid } & \multicolumn{3}{c}{ Teicoplanin } & \multicolumn{3}{c}{ Daptomycin } & \multicolumn{2}{c}{ Tigecyclin } \\
Organism & S & I & R & S & I & R & S & I & R & S & I & R & S & I & R \\
S.aureus & $\leq 2$ & $4-8$ & $\geq 16$ & $\leq 4$ & - & $\geq 8$ & $\leq 8$ & 16 & $\geq 32$ & $\leq 1$ & - & - & $\leq 0.5$ & - & $\geq 8$ \\
S.epidermidis & $\leq 4$ & $8-16$ & $\geq 32$ & $\leq 4$ & - & $\geq 8$ & $\leq 8$ & 16 & $\geq 32$ & $\leq 1$ & - & - & $\leq 0.5$ & - & $\geq 8$ \\
S.haemolyticus & $\leq 4$ & $8-16$ & $\geq 32$ & $\leq 4$ & - & $\geq 8$ & $\leq 8$ & 16 & $\geq 32$ & $\leq 1$ & - & - & $\leq 0.5$ & - & $\geq 8$ \\
Others & $\leq 4$ & $8-16$ & $\geq 32$ & $\leq 4$ & - & $\geq 8$ & $\leq 8$ & 16 & $\geq 32$ & $\leq 1$ & - & - & $\leq 0.5$ & - & $\geq 8$ \\
\hline
\end{tabular}

n.b. S- Sensitive, I- Intermidiate, R-Resistant

Table 2: Species distribution of various clinically significant Staphylococci

\begin{tabular}{|c|c|c|c|c|}
\hline & Total & Cef pos & $\%$ & Inducible Clindamycin resistance (\%) \\
\hline S.aureus (MRSA) & 16 & 16 & 41.03 & $37 \cdot 5$ \\
\hline MSSA & 23 & oo & oo & 39.1 \\
\hline S.epidermidis & 72 & 70 & 97.2 & 19.7 \\
\hline S.haemolyticus & 68 & 66 & 97.06 & 18.9 \\
\hline S.hominis & 46 & 46 & 100 & 15.2 \\
\hline S. lugdenensis & 06 & 06 & 100 & o \\
\hline S cohnii & 14 & 14 & 100 & o \\
\hline S capitis & 11 & 11 & 100 & o \\
\hline S auricularis & 01 & 01 & 100 & o \\
\hline S simulans & 01 & 01 & 100 & o \\
\hline Ssciuri & $\mathrm{O} 1$ & $\mathrm{O} 1$ & 100 & o \\
\hline S saprophyticus & 03 & 02 & 66.7 & o \\
\hline S warneri & $\mathrm{O} 2$ & 01 & 50 & o \\
\hline
\end{tabular}

Table 3: Sensitivity pattern (in \%) of the different species of Staphylococcus

\begin{tabular}{|c|c|c|c|c|c|c|c|c|c|}
\hline & MSSA (23) & MRSA (16) & $\begin{array}{l}\text { SE } \\
(72)\end{array}$ & $\begin{array}{l}\text { SH } \\
(67)\end{array}$ & $\begin{array}{c}\text { SHom } \\
(46)\end{array}$ & $\begin{array}{l}\text { SC } \\
(14)\end{array}$ & $\begin{array}{l}\text { SL } \\
(6)\end{array}$ & $\begin{array}{l}\text { Scap } \\
\text { (11) }\end{array}$ & $\begin{array}{c}\text { Others } \\
(7)\end{array}$ \\
\hline $\mathbf{P}$ & 30.4 & 13.3 & 12.67 & 6.15 & 17.4 & 21.4 & $33 \cdot 3$ & 9.09 & o \\
\hline $\mathbf{O x}$ & 95.6 & 18.75 & 18.3 & 6.15 & 10.9 & $27 \cdot 3$ & 50 & 27.3 & 100 \\
\hline Gen & 91.3 & 68.7 & 83.1 & 36.5 & $95 \cdot 7$ & 78.6 & 100 & 81.8 & 100 \\
\hline Cip & 13.04 & 25.0 & 45.07 & $15 \cdot 38$ & 60.9 & 71.4 & 100 & $54 \cdot 5$ & $85 \cdot 7$ \\
\hline Le & 13.04 & 18.75 & 43.66 & $15 \cdot 38$ & 60.9 & 71.4 & 100 & $54 \cdot 5$ & $85 \cdot 7$ \\
\hline $\mathbf{E}$ & 26.08 & $43 \cdot 75$ & 21.13 & 18.97 & 32.6 & $64 \cdot 3$ & 100 & $45 \cdot 5$ & 28.6 \\
\hline CLD & 60.9 & 56.25 & 52.11 & 34.84 & 60.9 & $64 \cdot 3$ & 100 & $45 \cdot 5$ & 42.9 \\
\hline Te & $95 \cdot 6$ & $93 \cdot 7$ & 88.7 & 89.29 & $95 \cdot 7$ & 100 & 100 & 72.7 & $85 \cdot 7$ \\
\hline Nit & 100 & 100 & 100 & 96.43 & 100 & 100 & 100 & 100 & 100 \\
\hline Rif & 100 & 100 & 100 & 80.36 & 100 & 85.7 & 100 & 81.8 & 85.7 \\
\hline Cot & 91.3 & 68.75 & 71.2 & 80.36 & $78 \cdot 3$ & 78.6 & 100 & 90.9 & 71.4 \\
\hline $\mathbf{L z}$ & 100 & 100 & 88.7 & 81.54 & 100 & $85 \cdot 7$ & 100 & 90.9 & 100 \\
\hline $\mathbf{V}$ & 100 & 100 & 100 & 100 & 100 & 100 & 100 & 100 & 100 \\
\hline Dap & 100 & 100 & 98.59 & 98.39 & 97.8 & 78.6 & 100 & 100 & 100 \\
\hline Tei & 100 & 100 & 92.95 & $95 \cdot 34$ & 91.3 & 100 & 100 & 100 & 100 \\
\hline Tgc & 95.6 & 100 & 100 & 100 & 100 & 100 & 100 & 100 & 100 \\
\hline
\end{tabular}




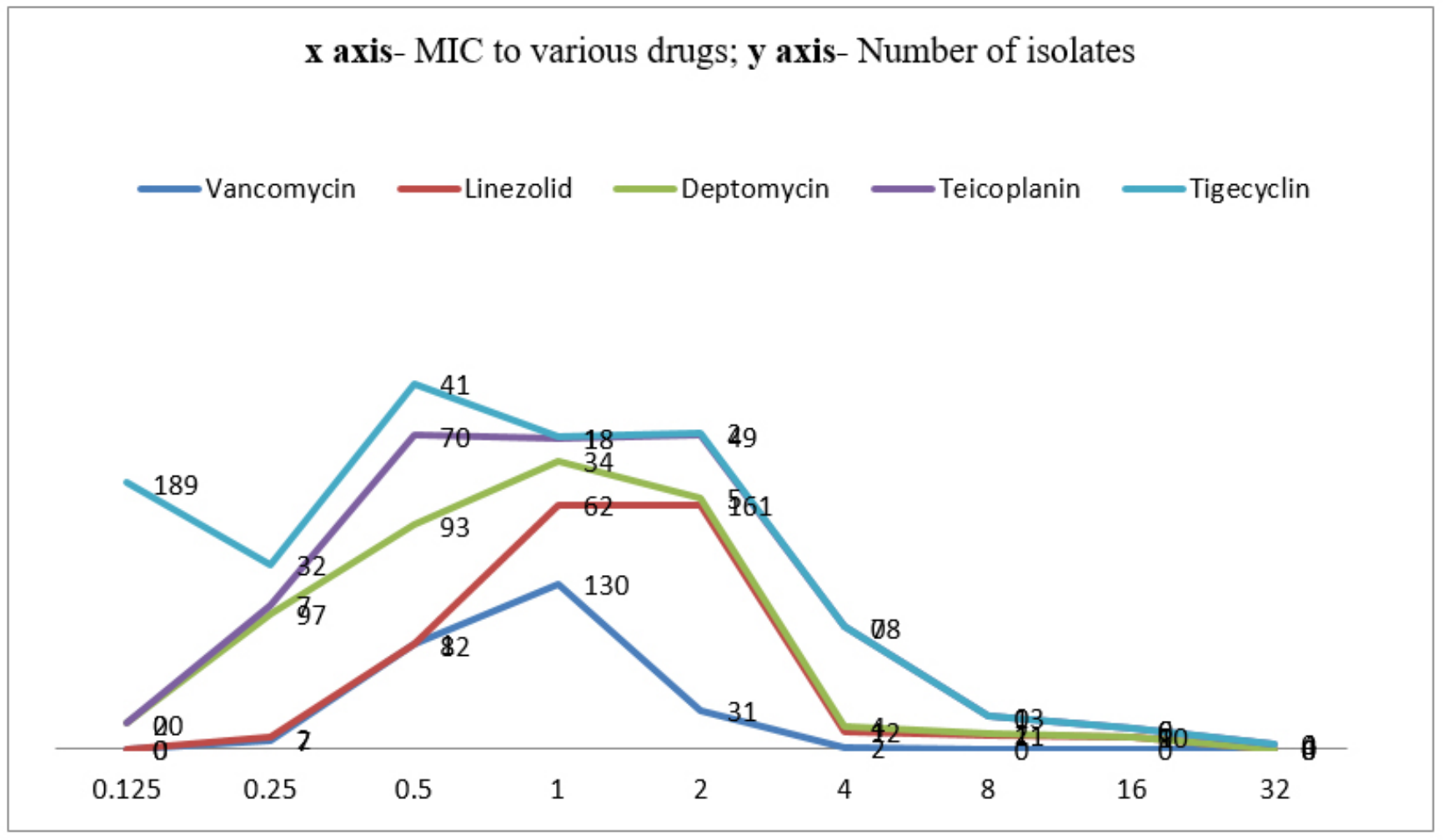

Figure 1: The MIC of Staphylococci to various drugs.

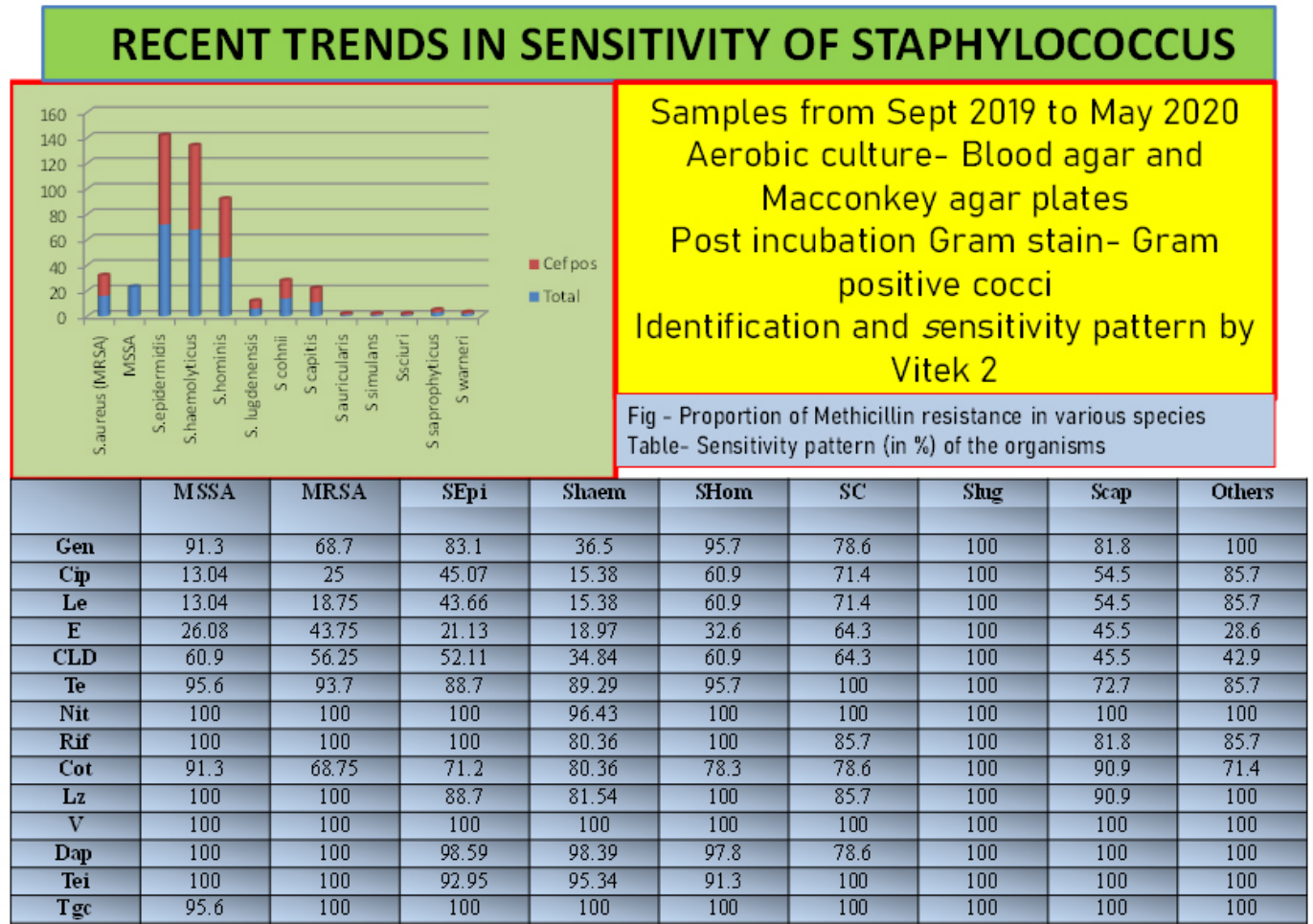

